\title{
The association between consumption of monounsaturated fats from animal- $v$. plant-based foods and the risk of type 2 diabetes: a prospective nationwide cohort study
}

\author{
Pan Zhuang ${ }^{1}$, Yu Zhang ${ }^{1}$, Lei $\mathrm{MaO}^{2}$, Liang Wang ${ }^{3}$, Fei Wu² ${ }^{2}$ Longfei Cheng ${ }^{2}$ and Jingjing Jiao ${ }^{4 *}$ \\ ${ }^{1}$ National Engineering Laboratory of Intelligent Food Technology and Equipment, Zhejiang Key Laboratory for Agro-Food \\ Processing, College of Biosystems Engineering and Food Science, Zhejiang University, Hangzhou, People's Republic of China \\ ${ }^{2}$ Department of Nutrition, School of Public Health, Zhejiang University School of Medicine, Hangzhou, People's Republic of China \\ ${ }^{3}$ Department of Biostatistics and Epidemiology, College of Public Health, East Tennessee State University, Johnson City, TN, USA \\ ${ }^{4}$ Department of Nutrition, School of Public Health, and Department of Nutrition of Affiliated Second Hospital, Zhejiang \\ University School of Medicine, Hangzhou, People's Republic of China
}

(Submitted 11 September 2019 - Final revision received 17 January 2020 - Accepted 10 February 2020 - First published online 27 February 2020 )

\section{Abstract}

Although higher dietary intake of MUFA has been shown to improve glycaemic control and lipid profiles, whether MUFA consumption from different sources is linked to the development of type 2 diabetes (T2D) remains unclear. We aimed to prospectively assess the associations of plant-derived MUFA (P-MUFA) and animal-derived MUFA (A-MUFA) intakes with T2D risk in a nationwide oriental cohort. Overall, 15022 Chinese adults, aged $\geq 20$ years, from the China Health and Nutrition Survey (CHNS 1997-2011) were prospectively followed up for a median of 14 years. Consumption of MUFA from plant and animal sources was assessed using 3-d 24-h recalls in each survey, and the cumulative average of intake was calculated. Multivariable-adjusted Cox models were constructed to estimate the hazard ratios (HR) of T2D according to quartiles of MUFA intake. P-MUFA were mainly consumed from cooked vegetable oils, fried bread sticks and rice, while A-MUFA were mainly consumed

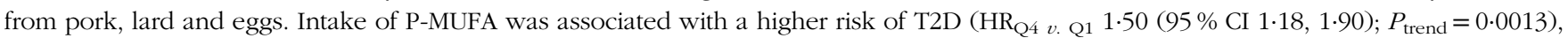
whereas A-MUFA showed no significant association $\left(\mathrm{HR}_{\mathrm{Q} 4} v\right.$. Q1 $\left.0.84(95 \% \mathrm{CI} 0.59,1 \cdot 20) ; P_{\text {trend }}=0.30\right)$. When further considering the cooking method of food sources, consumption of P-MUFA from fried foods was positively associated with T2D risk (HR $\mathrm{Q}_{4} v$. Q1 1.60 (95\% CI 1.26, 2.02); $P_{\text {trend }}=0.0006$ ), whereas non-fried P-MUFA were not associated. Intake of MUFA from fried plant-based foods may elevate T2D risk among the Chinese population.

Key words: MUFA: Plant sources of fat: Animal sources of fat: Type 2 diabetes: China Health and Nutrition Survey: Nationwide cohorts

The prevalence of type 2 diabetes (T2D) has increased rapidly in both developed and developing countries over the last few decades. The number of T2D incidences has globally reached 425 million ( $8.8 \%$ of adults) with an additional 352 million at risk of developing $\mathrm{T}_{2} \mathrm{D}^{(1)}$. Typically, the morbidity of $\mathrm{T} 2 \mathrm{D}$ in China sharply increased from $2.5 \%$ in $1994^{(2)}$ to $10 \cdot 9 \%$ in $2013^{(3)}$, which imposes a substantial disease toll and economic burden to patients and healthcare systems.

Epidemiological evidence has consistently demonstrated that diet is a key modifiable risk factor for T2D prevention ${ }^{(4)}$. Although prevailing dietary guidelines advocate substituting SFA with unsaturated fatty acids, including MUFA and PUFA, the recommendation is mainly based on the cardiovascular benefit and less is known about the effects of dietary unsaturated fatty acids, especially MUFA, on T2D prevention. Multiple studies showed that the Mediterranean diet improved glycaemic control, insulin sensitivity and lipid profiles probably due to the intakes of MUFA and PUFA ${ }^{(5)}$. Meta-analyses of randomised controlled trials demonstrated that high-MUFA diets were effective in reducing glycated $\mathrm{Hb}$ (HbA1c) among individuals with glucose metabolism disorders ${ }^{(6)}$ and improving metabolic risk factors including fasting plasma glucose among T2D patients ${ }^{(7)}$. However, little evidence supported the beneficial effect of MUFA on glucose metabolism among healthy individuals ${ }^{(8,9)}$.

Abbreviations: A-MUFA, animal-derived MUFA; CHNS, China Health and Nutrition Survey; HR, hazard ratio; OA, oleic acid; PA, palmitoleic acid; P-MUFA, plantderived MUFA; T2D, type 2 diabetes.

* Corresponding author: Jingjing Jiao, email jingjingjiao@zju.edu.cn 
The associations of MUFA consumption with the risk of T2D development remain limited and controversial. Recently, the Tehran Lipid and Glucose Study found the intake of total MUFA was inversely associated with T2D incidence ${ }^{(10)}$, whereas most of the previous cohort studies in European/American countries reported non-significant associations ${ }^{(11-16)}$. A positive relationship between MUFA intake and T2D risk was even observed in the Australian Longitudinal Study on Women's Health $^{(17)}$. These inconsistent findings might be ascribed to the MUFA from different food sources that have divergent health benefits. Dietary MUFA from plant-based foods, such as nonhydrogenated vegetable oils and nuts, may be protective against T2D ${ }^{(14)}$. For example, olive oil consumption was overall associated with a lower risk of $\mathrm{T} 2 \mathrm{D}^{(18)}$. In contrast, consumption of MUFA from animal sources such as meat and dairy products was accompanied by high consumption of SFA, which could confound the relationship between total MUFA consumption and risk of $\mathrm{T} 2 \mathrm{D}^{(19)}$. Therefore, separating MUFA from plant and animal sources is needed to elucidate the role of MUFA in T2D development. China has one-fourth of the world's diabetes patients, which will reach 120 million in $2045^{(1)}$. However, the association of MUFA intake with T2D incidence has not been reported in the Chinese population. Therefore, we aimed to separate MUFA from animal and plant sources and prospectively assessed their long-term associations with T2D risk in the China Health and Nutrition Survey (CHNS) consisting of a nationwide oriental population.

\section{Materials and methods}

\section{Study population}

The CHNS is an ongoing, large-scale, longitudinal and householdbased cohort initiated in 1989 to assess the relationships between the transformation of society and the economy and the resulting health and nutritional status of the Chinese population ${ }^{(20)}$. The survey has been successfully conducted in nine rounds (1989, 1991, 1993, 1997, 2000, 2004, 2006, 2009 and 2011), which was approved by the institutional review committees of the University of North Carolina at Chapel Hill and the National Institute of Nutrition and Food Safety at Chinese Center for Disease Control and Prevention. Since no information on T2D diagnosis was collected before 1997, data from six rounds thereafter were utilised for present analysis. A total of 26889 participants were eligible between the 1997 and 2011 surveys. We excluded the participants aged $<20$ years at entry ( $n$ 6856) and those without complete dietary data ( $n$ 4549). After further excluding those with implausible energy intake $(<3350$ or $>17570 \mathrm{~kJ} / \mathrm{d}$ for men and $<2510$ or $>14640 \mathrm{~kJ} / \mathrm{d}$ for women, $n$ 78 ), prevalent T2D cases ( $n$ 252), myocardial infarction or stroke ( $n$ 132) at baseline, a total of 15022 participants ( 7035 men and 7987 women) were enrolled in the final analyses of the present study (online Supplementary Fig. S1).

\section{Dietary assessment and covariates}

Dietary intakes for individual participants were assessed using the daily interview to collect the information on at-home food consumption over three consecutive days and asking the participants to report all foods consumed away from home on a 24-h recall basis over the same periods. Household data on food consumption were also obtained using a weighing inventory during the same three consecutive days. Detailed procedures of dietary data collection have been described elsewhere ${ }^{(21,22)}$. Dietary intakes of nutrients were calculated using corresponding versions of Chinese Food Composition Table ${ }^{(23-25)}$ for each round to track changes of nutrient composition in foods over a longterm period. Three-day average intakes of dietary protein, carbohydrate and fats including MUFA, PUFA and SFA in each round were calculated, and then cumulative averages of the intakes in all available rounds from entry to the diagnosis of T2D or the end of follow-up were further calculated to represent long-term diet and minimise within-person variation. For example, if a person entered the CHNS in 1997 and was diagnosed with diabetes in 2005, then the cumulative average of MUFA intake is the sum of MUFA intakes in 1997, 2000 and 2004 divided by three. We also separated MUFA into plant-derived MUFA (P-MUFA) and animal-derived MUFA (A-MUFA) according to different food sources. P-MUFA were from plant-based foods, such as vegetable oils, rice, bread, vegetables, fruits, legumes and nuts. A-MUFA were MUFA from animal foods, including animal fats for cooking, red meat, eggs, poultry, fish and dairy products. For a few mixed-food items, ingredients were identified according to the recipes for home-prepared items or product labels for manufactured foods. We also separated MUFA into fried MUFA and non-fried MUFA according to the cooking method used for MUFA-containing foods. Fried MUFA were derived from stirfried, griddled and deep-fried foods, while non-fried MUFA were derived from boiled, steamed, raw and other non-fried foods. Intakes of oleic acid (OA) and palmitoleic acid (PA), two main subtypes of MUFA, were also calculated. In a validation study, the total energy intake calculated by the dietary assessment method in the CHNS was correlated with the total energy expenditure measured by the doubly labelled water method $(r 0.56, P<0.01 \text { for men; } r 0.60, P<0.01 \text { for women })^{(26)}$. Data on other demographic and lifestyle factors were also collected, including age, weight and height, education level, marital status, income, smoking, alcohol drinking, physical activity, history of hypertension, geographical location (south or north) and site (urban or rural) ${ }^{(20)}$.

\section{Ascertainment of diabetes}

Diabetes status was identified by the questionnaire inquiring whether the person had physician-diagnosed T2D. Detailed information on the diagnosis date and diabetes treatment, including the special diet, weight control, oral medication, insulin and Chinese traditional medicine, was collected to reconfirm the self-reported cases in each round interview. In the 2009 survey, overnight fasting blood samples of participants were collected, while plasma glucose and HbA1c levels were analysed with strict quality control. The participants with a fasting plasma glucose $\geq 7.0 \mathrm{mmol} / \mathrm{l}$ or $\mathrm{HbA} 1 \mathrm{c} \geq 6.5 \%$ were then defined as diabetes in addition to self-reported diabetes based on the questionnaire. Among 15022 individuals in the present analysis, $7708(51.3 \%)$ had available plasma glucose data collected in the 
2009 round. A cross-sectional analysis of 10215 participants in the 2009 survey was conducted to evaluate the specificity of self-reported diabetes. Of 9964 participants self-reporting no diabetes, 753 participants $(7.6 \%)$ were diagnosed with diabetes according to the levels of fasting plasma glucose and HbA1c. Thus, $92.4 \%$ of participants who did not report diagnosed diabetes were below the blood glucose threshold for diabetes, indicating a low rate of undiagnosed diabetes in this population.

\section{Statistical analysis}

The intakes of protein, carbohydrate and fat were expressed as the percentages of total energy using the nutrient density method and divided into quartiles with the first quartile as the reference for analyses. Person-years of the follow-up were calculated from the round at entry to the year when participants were diagnosed with T2D, year of death or the year 2011, whichever came first. A median of 14 years of follow-up (mean 11.2 (SD $4 \cdot 2$ ) years; range 1-14 years) was documented for this study population. Hazard ratios (HR) and $95 \%$ CI for T2D were estimated using Cox proportional hazards regression models with follow-up duration (years) as the timescale. The end point of T2D diagnosis was coded as 1 , and censoring at death or not having diabetes during the follow-up was coded as 0 . The multivariate model was adjusted for established and potential risk factors for T2D, including age, sex, BMI, education, marital status, income, physical activity, smoking, alcohol drinking status, history of hypertension, north-south position (north or south), site (urban or rural), intake of total energy, percentage of energy from dietary protein, SFA and PUFA. The comparison macronutrient for MUFA was total carbohydrates in this model because the increase in dietary fat consumption was often simultaneously accompanied by the reduction in carbohydrate consumption in practice. We created indicator variables for missing data in each covariate, if necessary. Standard errors and variance of the estimates were also adjusted by clustering at the community level to control for design effects. Tests for trend were computed using the median values of each category of MUFA as continuous variables in the regression models.

We also assessed the change of T2D risk for isoenergetically replacing SFA with MUFA by establishing the substitution models that included total energy, protein, carbohydrate, MUFA and PUFA. In an isoenergetic setting, total energy intake and all other macronutrient intakes are held constant, such that the reduction of one macronutrient as a percentage of total energy intake will be compensated by the same percentage of increment of energy from another macronutrient ${ }^{(27)}$. When leaving SFA out of the isoenergetic model, the regression coefficients for MUFA bear the interpretation as the theoretical effect of substituting MUFA for the same amount of energy from SFA while holding the other nutrient intakes constant. Data on MUFA intake were continuous in this substitution model to estimate effects of SFA replacement by a fixed percentage of energy (5\%). Given the fact that A-MUFA and SFA derived from common food sources, the changes in risk of T2D by substituting P-MUFA for the sum of A-MUFA and SFA were also estimated.

Sensitivity analyses were also conducted to verify the robustness of our findings. We excluded persons who developed incident T2D during the initial 3 years of follow-up, further adjusted for fruit and vegetable consumption or dietary cholesterol intake to observe whether the results materially changed. Additionally, an alternative healthy eating index (2010) ${ }^{(28)}$ was further adjusted to see whether the results were explained by the overall dietary pattern. We also restricted our analysis to participants with both blood glucose data and self-reported information on T2D ( $n$ 7708) to eliminate the possibility of underestimation of the cases caused by self-reported T2D alone. Subgroup analyses were also conducted according to important potential effect modifiers and stratified by age, sex, BMI, smoking status, alcohol consumption, physical activity, north-south position, site and individual income. $P$ values for interactions were tested by likelihood-ratio test.

Statistical analyses were performed using the SAS statistical package (version 9.4; SAS Institute). Statistical tests were two-sided, and significance was defined as $P<0.05$.

\section{Results \\ Characteristics of the population from the China Health and Nutrition Survey}

The baseline characteristics of the participants by quartiles of total MUFA consumption are shown in Table 1. Participants with higher intakes of MUFA, P-MUFA and A-MUFA were on average older, more often urban residents and southerners, more educated, less physically active and tended to consume more SFA and vegetables but fewer carbohydrates. Moreover, individuals with higher P-MUFA consumption tended to be female, fatter, non-smokers and non-alcohol drinkers, and earned less money and consumed more PUFA but less protein. Participants with higher intake of A-MUFA were more likely to be male, wealthier, alcohol drinkers and consumed more protein and fruits.

\section{Consumption of MUFA from animal and plant sources}

In 1997-2011, the average intake of P-MUFA increased from 5.9 to $8.4 \%$ of energy, while intake of A-MUFA ranged from 3.6 to $8.6 \%$ (Fig. 1). Pork was the largest contributor of MUFA (21.6$38.9 \%$ of total MUFA) from animal sources (online Supplementary Table S1), followed by lard, eggs, pork chops, pork side ribs and duck. P-MUFA were mainly consumed from vegetable oils, including peanut oil ( $4 \cdot 3-17 \cdot 8 \%$ of total MUFA), rapeseed oil, soyabean oil, salad oil and tea-seed oil. Additionally, fried bread sticks and rice were also contributors to P-MUFA. For the individual MUFA, the food items contributing to OA were similar to those for total MUFA, while PA mainly came from pork, eggs, other animal products and soyabean oil.

The intake of P-MUFA was weakly correlated with the intake of A-MUFA $(r-0.05, P<0.0001$; online Supplementary Table S2). PUFA consumption was moderately correlated with P-MUFA consumption $(r$ 0.51, $P<0.0001)$, whereas it was weakly correlated with A-MUFA consumption ( $r$ 0.05, $P<0.0001)$. The correlation coefficient was high between SFA and A-MUFA consumption $(r 0 \cdot 86, P<0 \cdot 0001)$ but was low between SFA and P-MUFA consumption $(r 0 \cdot 28, P<0 \cdot 0001)$. 
N British Journal of Nutrition

Table 1. Baseline characteristics and dietary consumption of participants in the China Health and Nutrition Survey by quartiles (Q) of MUFA consumption ( $n$ 15022) (Percentages; mean values with their standard errors)

\begin{tabular}{|c|c|c|c|c|c|c|c|c|c|c|c|c|}
\hline \multirow[b]{2}{*}{ Characteristics } & \multicolumn{4}{|c|}{ Quartile of MUFA intake } & \multicolumn{4}{|c|}{ Quartile of P-MUFA intake } & \multicolumn{4}{|c|}{ Quartile of A-MUFA intake } \\
\hline & Q1 & Q2 & Q3 & Q4 & Q1 & Q2 & Q3 & Q4 & Q1 & Q2 & Q3 & Q4 \\
\hline Range (percentage of energy) & $\leq 9 \cdot 3$ & $9 \cdot 3-12 \cdot 7$ & $12 \cdot 7-16 \cdot 3$ & $\geq 16 \cdot 3$ & $\leq 4 \cdot 1$ & $4 \cdot 1-5 \cdot 9$ & $5 \cdot 9-8 \cdot 1$ & $\geq 8.1$ & $\leq 3 \cdot 4$ & $3 \cdot 4-6 \cdot 1$ & $6 \cdot 1-9 \cdot 0$ & $\geq 9 \cdot 0$ \\
\hline Male (\%) & 48.8 & $47 \cdot 0$ & $47 \cdot 2$ & $44 \cdot 3$ & $52 \cdot 3$ & 49.0 & $45 \cdot 8$ & $40 \cdot 3$ & $45 \cdot 1$ & $45 \cdot 6$ & 47.4 & 49.2 \\
\hline \multicolumn{13}{|l|}{ Age (years) } \\
\hline Mean & 40.7 & $42 \cdot 7$ & 43.7 & $45 \cdot 2$ & $40 \cdot 3$ & 41.9 & $44 \cdot 3$ & 45.8 & $42 \cdot 2$ & 43.0 & 43.6 & 43.4 \\
\hline SE & 0.2 & 0.2 & 0.2 & 0.3 & 0.2 & 0.2 & 0.2 & 0.3 & 0.2 & 0.2 & 0.2 & 0.3 \\
\hline \multicolumn{13}{|l|}{ BMI $\left(\mathrm{kg} / \mathrm{m}^{2}\right)$} \\
\hline Mean & 22.5 & 22.6 & $22 \cdot 7$ & $22 \cdot 7$ & 22.5 & $22 \cdot 6$ & $22 \cdot 6$ & $22 \cdot 8$ & 22.5 & $22 \cdot 7$ & $22 \cdot 8$ & $22 \cdot 6$ \\
\hline SE & 0.1 & 0.1 & 0.1 & 0.1 & 0.1 & 0.1 & 0.1 & 0.1 & 0.1 & $0 \cdot 1$ & 0.1 & 0.1 \\
\hline Married (\%) & 79.9 & $81 \cdot 4$ & $82 \cdot 1$ & 79.4 & $76 \cdot 7$ & 81.6 & 82.9 & 81.7 & 80.6 & $82 \cdot 7$ & 82.0 & $77 \cdot 6$ \\
\hline ZHigh school (\%) & $15 \cdot 3$ & $23 \cdot 3$ & 30.4 & 34.7 & $23 \cdot 1$ & 24.8 & $27 \cdot 7$ & 28.0 & $13 \cdot 6$ & 23.7 & 30.5 & 35.7 \\
\hline Vigorous activity level (\%) & $52 \cdot 2$ & 37.0 & $28 \cdot 1$ & $20 \cdot 6$ & 39.0 & $37 \cdot 7$ & $31 \cdot 6$ & 29.6 & $52 \cdot 7$ & 37.0 & $27 \cdot 7$ & $20 \cdot 4$ \\
\hline Current smoker (\%) & $22 \cdot 7$ & $21 \cdot 6$ & 20.0 & $21 \cdot 2$ & $23 \cdot 7$ & $22 \cdot 3$ & 20.5 & 18.9 & 21.4 & $20 \cdot 8$ & 21.0 & $22 \cdot 2$ \\
\hline Alcohol drinker (\%) & $22 \cdot 9$ & $25 \cdot 2$ & $25 \cdot 9$ & $27 \cdot 3$ & $26 \cdot 1$ & $26 \cdot 3$ & $25 \cdot 2$ & 23.8 & $21 \cdot 3$ & $25 \cdot 1$ & $27 \cdot 2$ & $27 \cdot 7$ \\
\hline History of hypertension (\%) & $10 \cdot 1$ & 10.8 & 9.8 & $10 \cdot 2$ & $10 \cdot 0$ & 10.4 & 10.2 & $10 \cdot 3$ & $10 \cdot 1$ & $10 \cdot 8$ & 10.7 & $9 \cdot 2$ \\
\hline Urban site (\%) & $18 \cdot 1$ & 29.4 & $41 \cdot 8$ & $57 \cdot 4$ & $30 \cdot 3$ & 32.0 & 38.8 & $45 \cdot 7$ & $17 \cdot 1$ & $32 \cdot 2$ & 41.8 & 55.7 \\
\hline North (\%) & 65.9 & $48 \cdot 6$ & $37 \cdot 6$ & $23 \cdot 7$ & 49.6 & $49 \cdot 3$ & $43 \cdot 2$ & 33.8 & $65 \cdot 2$ & $49 \cdot 3$ & 37.8 & $23 \cdot 7$ \\
\hline \multicolumn{13}{|l|}{ Individual income* } \\
\hline Mean & 6222 & 8523 & 8984 & 8836 & 7602 & 6661 & 6558 & 6340 & 4524 & 6856 & 7988 & 7790 \\
\hline SE & 166 & 248 & 196 & 282 & 283 & 179 & 148 & 139 & 113 & 184 & 200 & 254 \\
\hline \multicolumn{13}{|l|}{ Dietary intake } \\
\hline \multicolumn{13}{|l|}{ Total energy $(\mathrm{kcal} / \mathrm{d}) \dagger$} \\
\hline Mean & 2110 & 2069 & 2157 & 2825 & 2131 & 2130 & 2170 & 2730 & 2591 & 2119 & 2131 & 2319 \\
\hline SE & 8 & 8 & 8 & 165 & 9 & 8 & 8 & 165 & 165 & 9 & 8 & 10 \\
\hline \multicolumn{13}{|c|}{ Total carbohydrates (percentage of energy) } \\
\hline Mean & 68.9 & 60.5 & 54.1 & 44.6 & $63 \cdot 1$ & 59.6 & 55.9 & 49.5 & 65.8 & 59.5 & 55.0 & 47.9 \\
\hline SE & 0.1 & $0 \cdot 1$ & 0.1 & 0.1 & 0.2 & 0.1 & 0.1 & 0.2 & 0.2 & 0.1 & 0.1 & 0.1 \\
\hline \multicolumn{13}{|c|}{ Total protein (percentage of energy) } \\
\hline Mean & $12 \cdot 4$ & $12 \cdot 6$ & $12 \cdot 6$ & $11 \cdot 6$ & $12 \cdot 9$ & $12 \cdot 6$ & 12.4 & 11.4 & 11.6 & $12 \cdot 4$ & $12 \cdot 8$ & $12 \cdot 4$ \\
\hline SE & 0.03 & 0.04 & 0.04 & 0.04 & 0.0 & 0.0 & 0.0 & 0.0 & 0.0 & 0.0 & 0.0 & 0.0 \\
\hline \multicolumn{13}{|l|}{ SFA (percentage of energy) } \\
\hline Mean & 4.8 & 7.5 & 9.7 & $12 \cdot 9$ & 7.8 & $8 \cdot 2$ & 8.9 & $10 \cdot 1$ & $5 \cdot 3$ & $7 \cdot 4$ & $9 \cdot 3$ & $12 \cdot 8$ \\
\hline SE & 0.02 & 0.02 & 0.03 & 0.05 & 0.1 & 0.1 & 0.1 & 0.1 & 0.0 & 0.0 & 0.0 & 0.0 \\
\hline \multicolumn{13}{|l|}{ PUFA (percentage of energy) } \\
\hline Mean & $7 \cdot 1$ & $8 \cdot 3$ & 9.2 & $10 \cdot 7$ & $6 \cdot 3$ & $8 \cdot 2$ & $9 \cdot 3$ & 11.5 & 8.8 & 8.9 & $8 \cdot 8$ & $8 \cdot 7$ \\
\hline SE & 0.05 & 0.06 & 0.06 & 0.09 & 0.0 & 0.0 & 0.1 & 0.1 & 0.1 & 0.1 & 0.1 & 0.1 \\
\hline \multicolumn{13}{|l|}{ Fruits $(g / d)$} \\
\hline Mean & 36.5 & $46 \cdot 3$ & $50 \cdot 0$ & 43.2 & $46 \cdot 6$ & $45 \cdot 0$ & 44.6 & 39.8 & 28.5 & $48 \cdot 1$ & 52.5 & $46 \cdot 8$ \\
\hline SE & $1 \cdot 3$ & $1 \cdot 3$ & 1.5 & 1.4 & 1.5 & 1.3 & 1.4 & 1.3 & $1 \cdot 2$ & 1.4 & 1.4 & 1.5 \\
\hline \multicolumn{13}{|l|}{ Vegetables (g/d) } \\
\hline Mean & $476 \cdot 3$ & $450 \cdot 5$ & 464.5 & $515 \cdot 7$ & $467 \cdot 7$ & 469.7 & $480 \cdot 0$ & 489.7 & $492 \cdot 9$ & $447 \cdot 7$ & $448 \cdot 5$ & 517.9 \\
\hline SE & 4.3 & 3.8 & 3.7 & 4.0 & $4 \cdot 2$ & 4.0 & 3.7 & 3.9 & $4 \cdot 3$ & 3.8 & 3.5 & 4.1 \\
\hline
\end{tabular}

Individual income was inflated to 2009.

† To convert kcal to kJ, multiply by 4.184 . 


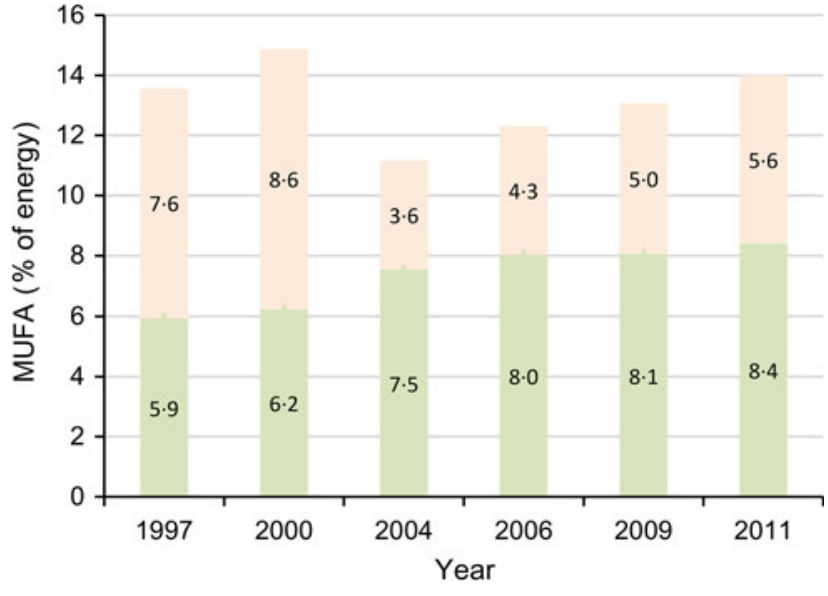

Fig. 1. Trends in animal-derived MUFA (A-MUFA) and plant-derived MUFA (P-MUFA) intakes (\% of energy) during a median of 14 years of follow-up. The numbers of enrolled participants were $12844,13562,11779,11284$, 11600 and 14996 in the 1997, 2000, 2004, 2006, 2009 and 2011 rounds in the China Health and Nutrition Survey, respectively. , A-MUFA; , P-MUFA.

\section{Plant-derived MUFA, animal-derived MUFA and risk of} type 2 diabetes

During a total of 167698 person-years of follow-up, 1014 incident T2D cases were documented. Although the intake of total MUFA was not associated with T2D risk in the age- and sex-adjusted model, a positive association was observed after Health and Nutrition Survey

(Numbers and percentages; hazard ratios (HR) and $95 \%$ confidence intervals) adjusting for other confounders in the multivariable-adjusted model (Table 2). Compared with the participants with the lowest quartile of MUFA intake, those in the highest quartile had an $81 \%$ higher risk of T2D (HR 1.81; $95 \%$ CI 1.28, 2.57; $P_{\text {trend }}=0.0015$ ). With regard to MUFA from different sources, P-MUFA intake was associated with a progressively higher T2D risk. The HR for the increasing quartiles were 1.22 (95\% CI 1.00, 1.50), 1.27 (95\% CI $1.02,1.57)$ and $1.50(95 \%$ CI $1.18,1.90)\left(P_{\text {trend }}=0.0013\right)$. However, A-MUFA did not show any association with T2D risk $\left(P_{\text {trend }}=0 \cdot 30\right)$

\section{MUFA from fried and non-fried foods and risk of type 2 diabetes}

Consumption of MUFA from fried foods was strongly associated with a higher T2D risk $\left(P_{\text {trend }}=0.0001\right)$, which was driven by the positive association of fried P-MUFA with T2D risk $\left(P_{\text {trend }}=0.0006\right.$ ) (Table 3). Multivariable HR comparing extreme quartiles of intake were 1.74 (95\% CI 1.31, 2.30) for fried MUFA and $1.60(95 \%$ CI 1.26, 2.02) for fried P-MUFA. Intakes of fried A-MUFA and non-fried MUFA, including non-fried P-MUFA and non-fried A-MUFA, were all not associated with the risk of T2D.

\section{Oleic acid, palmitoleic acid and risk of type 2 diabetes}

For individual MUFA, we observed a positive relationship between $\mathrm{OA}$ intake and T2D risk, whereas a negative association was detected between PA intake and T2D risk $\left(P_{\text {trend }}<0 \cdot 0001\right)$

Table 2. Associations between MUFA, plant-derived MUFA (P-MUFA) and animal-derived MUFA (A-MUFA) intakes and type 2 diabetes risk in the China

\begin{tabular}{|c|c|c|c|c|c|}
\hline & \multicolumn{4}{|c|}{ Quartiles of MUFA intake } & \multirow[b]{2}{*}{$P_{\text {trend }}$} \\
\hline & Q1 & Q2 & Q3 & Q4 & \\
\hline \multicolumn{6}{|l|}{ Total MUFA } \\
\hline Range (percentage of energy) & $\leq 9 \cdot 3$ & $9 \cdot 3-12 \cdot 7$ & $12 \cdot 7-16 \cdot 3$ & $\geq 16 \cdot 3$ & \\
\hline No. of cases & 221 & 261 & 278 & 254 & \\
\hline$\%$ & $5 \cdot 9$ & $7 \cdot 0$ & $7 \cdot 4$ & $6 \cdot 8$ & \\
\hline Age- and sex-adjusted HR & 1.00 & $1 \cdot 14$ & $1 \cdot 11$ & $0 \cdot 88$ & 0.079 \\
\hline $95 \% \mathrm{Cl}$ & & $0.95,1.36$ & $0.93,1.32$ & $0.73,1.05$ & \\
\hline Multivariable-adjusted $\mathrm{HR}^{*}$ & 1.00 & $1 \cdot 36$ & 1.50 & 1.81 & 0.0015 \\
\hline $95 \% \mathrm{Cl}$ & & $1.06,1.73$ & $1 \cdot 12,2 \cdot 01$ & $1 \cdot 28,2 \cdot 57$ & \\
\hline \multicolumn{6}{|l|}{ P-MUFA } \\
\hline Range (percentage of energy) & $\leq 4 \cdot 1$ & $4 \cdot 1-5 \cdot 9$ & $5 \cdot 9-8 \cdot 1$ & $\geq 8 \cdot 1$ & \\
\hline No. of cases & 171 & 255 & 288 & 300 & \\
\hline$\%$ & $4 \cdot 6$ & $6 \cdot 8$ & $7 \cdot 7$ & $8 \cdot 0$ & \\
\hline Age- and sex-adjusted HR & 1.00 & $1 \cdot 25$ & $1 \cdot 26$ & $1 \cdot 25$ & 0.078 \\
\hline $95 \% \mathrm{Cl}$ & & $1.03,1.51$ & $1.04,1.53$ & $1.03,1.51$ & \\
\hline Multivariable-adjusted $\mathrm{HR}^{*}$ & 1.00 & $1 \cdot 22$ & $1 \cdot 27$ & 1.50 & 0.0013 \\
\hline \multicolumn{6}{|l|}{ A-MUFA } \\
\hline Range (percentage of energy) & $\leq 3.4$ & $3 \cdot 4-6 \cdot 1$ & $6 \cdot 1-9 \cdot 0$ & $\geq 9.0$ & \\
\hline No. of cases & 250 & 284 & 283 & 197 & \\
\hline$\%$ & 6.7 & $7 \cdot 6$ & $7 \cdot 5$ & $5 \cdot 3$ & \\
\hline Age- and sex-adjusted HR & 1.00 & $1 \cdot 19$ & $1 \cdot 13$ & 0.72 & 0.0004 \\
\hline $95 \% \mathrm{Cl}$ & & $1.00,1.41$ & $0.96,1.34$ & $0.60,0.87$ & \\
\hline Multivariable-adjusted $\mathrm{HR}^{*}$ & 1.00 & $1 \cdot 11$ & 0.99 & $0 \cdot 84$ & 0.30 \\
\hline $95 \% \mathrm{Cl}$ & & $0.88,1.38$ & $0.75,1.32$ & $0.59,1.20$ & \\
\hline
\end{tabular}

*Adjusted for age, sex, BMI (in kg/m²; <18.5, 18.5-23.9, 24-27.9 or $\geq 28$ ), education (less than high school, high school, some college or at least college), marital status (never married, married or living as married, widowed/divorced/separated or unknown), income (quartile), north-south position (north or south), and site (urban or rural), physical activity (no regular activity, low to moderate activity or vigorous activity), smoking (never, former, current or unknown), alcohol drinking status (abstainer or drinker), history of hypertension (yes, no or unknown), intake of total energy, percentages of energy intake from protein, SFA, PUFA, and remaining MUFA where appropriate (P-MUFA and A-MUFA). 
Table 3. Associations of MUFA from fried and non-fried foods with type 2 diabetes risk in the China Health and Nutrition Survey (Numbers and percentages; hazard ratios (HR) and $95 \%$ confidence intervals)

\begin{tabular}{|c|c|c|c|c|c|}
\hline & \multicolumn{4}{|c|}{ Quartiles of MUFA intake } & \multirow[b]{2}{*}{$P_{\text {trend }}$} \\
\hline & Q1 & Q2 & Q3 & Q4 & \\
\hline \multicolumn{6}{|l|}{ Fried MUFA } \\
\hline Range (percentage of energy) & $\leq 5 \cdot 2$ & $5 \cdot 2-8 \cdot 0$ & $8 \cdot 0-11 \cdot 2$ & $\geq 11.2$ & \\
\hline No. of cases & 206 & 269 & 282 & 257 & \\
\hline$\%$ & 5.5 & $7 \cdot 2$ & 7.5 & $6 \cdot 8$ & \\
\hline Age- and sex-adjusted HR & 1.00 & 1.14 & 1.09 & 0.92 & 0.23 \\
\hline $95 \% \mathrm{Cl}$ & & $0.95,1.37$ & $0.91,1.31$ & $0.76,1 \cdot 10$ & \\
\hline Multivariable-adjusted $\mathrm{HR}^{\star}$ & 1.00 & 1.28 & 1.43 & 1.74 & 0.0001 \\
\hline $95 \% \mathrm{Cl}$ & & $1.05,1.56$ & $1 \cdot 14,1.79$ & $1 \cdot 31,2 \cdot 30$ & \\
\hline \multicolumn{6}{|l|}{ Fried P-MUFA } \\
\hline Range (percentage of energy) & $\leq 3 \cdot 1$ & $3 \cdot 1-4 \cdot 8$ & $4 \cdot 8-7 \cdot 1$ & $\geq 7 \cdot 1$ & \\
\hline No. of cases & 171 & 264 & 275 & 304 & \\
\hline$\%$ & 4.6 & $7 \cdot 0$ & $7 \cdot 3$ & $8 \cdot 1$ & \\
\hline Age- and sex-adjusted HR & 1.00 & 1.30 & 1.22 & 1.28 & 0.052 \\
\hline $95 \% \mathrm{Cl}$ & & $1.07,1.58$ & $1.00,1.47$ & $1.06,1.55$ & \\
\hline Multivariable-adjusted $\mathrm{HR}^{\star}$ & 1.00 & 1.33 & 1.26 & 1.60 & 0.0006 \\
\hline $95 \% \mathrm{Cl}$ & & $1.08,1.62$ & $1.02,1.56$ & $1 \cdot 26,2.02$ & \\
\hline \multicolumn{6}{|l|}{ Fried A-MUFA } \\
\hline Range (percentage of energy) & $\leq 0.8$ & $0.8-2 \cdot 4$ & $2.4-4.7$ & $\geq 4.7$ & \\
\hline No. of cases & 247 & 289 & 267 & 211 & \\
\hline$\%$ & $6 \cdot 6$ & $7 \cdot 7$ & $7 \cdot 1$ & 5.6 & \\
\hline Age- and sex-adjusted HR & 1.00 & 1.23 & 1.07 & 0.77 & 0.0017 \\
\hline $95 \% \mathrm{Cl}$ & & $1.03,1.45$ & $0.90,1.28$ & $0.64,0.92$ & \\
\hline Multivariable-adjusted $\mathrm{HR}^{\star}$ & 1.00 & 1.09 & 1.03 & 0.99 & 0.97 \\
\hline $95 \% \mathrm{Cl}$ & & $0.91,1.31$ & $0.84,1.27$ & $0.75,1.31$ & \\
\hline \multicolumn{6}{|l|}{ Non-fried MUFA } \\
\hline Range (percentage of energy) & $\leq 2 \cdot 0$ & $2.0-3.7$ & $3.7-5.9$ & $\geq 5.9$ & \\
\hline No. of cases & 210 & 244 & 324 & 236 & \\
\hline$\%$ & $5 \cdot 6$ & 6.5 & $8 \cdot 6$ & $6 \cdot 3$ & \\
\hline Age- and sex-adjusted HR & 1.00 & $1 \cdot 10$ & 1.53 & $1 \cdot 11$ & 0.025 \\
\hline $95 \% \mathrm{Cl}$ & & $0.92,1.32$ & $1.29,1.82$ & $0.92,1.33$ & \\
\hline Multivariable-adjusted $\mathrm{HR}^{*}$ & 1.00 & 0.96 & 1.26 & 1.12 & 0.11 \\
\hline $95 \% \mathrm{Cl}$ & & $0.79,1.17$ & $1.02,1.55$ & $0.86,1.45$ & \\
\hline \multicolumn{6}{|l|}{ Non-fried P-MUFA } \\
\hline Range (percentage of energy) & $\leq 0.6$ & $0.6-0.8$ & $0.8-1 \cdot 2$ & $\geq 1 \cdot 2$ & \\
\hline No. of cases & 229 & 255 & 258 & 272 & \\
\hline$\%$ & $6 \cdot 1$ & $6 \cdot 8$ & 6.9 & $7 \cdot 2$ & \\
\hline Age- and sex-adjusted HR & 1.00 & 0.94 & 0.95 & 1.06 & 0.47 \\
\hline $95 \% \mathrm{Cl}$ & & $0.78,1 \cdot 12$ & $0.79,1.13$ & $0.89,1.26$ & \\
\hline Multivariable-adjusted $\mathrm{HR}^{\star}$ & 1.00 & 0.91 & 0.91 & 0.90 & 0.30 \\
\hline $95 \% \mathrm{Cl}$ & & $0.76,1.09$ & $0.75,1.09$ & $0.74,1.08$ & \\
\hline \multicolumn{6}{|l|}{ Non-fried A-MUFA } \\
\hline Range (percentage of energy) & $\leq 1 \cdot 0$ & $1 \cdot 0-2 \cdot 6$ & $2 \cdot 6-4 \cdot 8$ & $\geq 4 \cdot 8$ & \\
\hline No. of cases (\%) & 206 & 258 & 318 & 232 & \\
\hline$\%$ & 5.5 & $6 \cdot 9$ & 8.5 & $6 \cdot 2$ & \\
\hline Age- and sex-adjusted HR & 1.00 & 1.24 & 1.54 & $1 \cdot 13$ & 0.049 \\
\hline $95 \% \mathrm{Cl}$ & & $1.04,1.49$ & $1.29,1.83$ & $0.94,1.36$ & \\
\hline Multivariable-adjusted $\mathrm{HR}^{\star}$ & 1.00 & 1.01 & 1.19 & 0.97 & 0.70 \\
\hline $95 \% \mathrm{Cl}$ & & $0.83,1.23$ & $0.96,1.48$ & $0.74,1.28$ & \\
\hline
\end{tabular}

P-MUFA, plant-derived MUFA; A-MUFA, animal-derived MUFA.

*Adjusted for age, sex, BMI (in kg/m²; <18.5, 18.5-23.9, 24-27.9 or $\geq 28$ ), education (less than high school, high school, some college or at least college), marital status (never married, married or living as married, widowed/divorced/separated or unknown), income (quartile), north-south position (north or south), and site (urban or rural), physical activity (no regular activity, low to moderate activity, or vigorous activity), smoking (never, former, current, or unknown), alcohol drinking status (abstainer or drinker), history of hypertension (yes, no, or unknown), intake of total energy, percentages of energy intake from protein, SFA, PUFA, and remaining MUFA where appropriate (fried MUFA, non-fried MUFA, fried P-MUFA, non-fried P-MUFA, fried A-MUFA and non-fried A-MUFA).

(online Supplementary Table S3). Multivariable HR comparing extreme quartiles were 2.35 (95\% CI 1.59, 3.47) for OA intake $\left(P_{\text {trend }}<0.0001\right)$ and $0.44(95 \%$ CI $0.32,0.60)$ for PA intake $\left(P_{\text {trend }}<0 \cdot 0001\right)$. Furthermore, the intakes of plant-derived OA and plant-derived PA were both correlated with a higher risk of T2D. Animal-derived PA intake was related to a lower risk of $\mathrm{T} 2 \mathrm{D}$, whereas the association for animal-derived OA (A-OA) was not observed $\left(P_{\text {trend }}=0 \cdot 50\right)$.

\section{Substitution for SFA}

The results of substitution analyses show that the HR for isoenergetically replacing $5 \%$ of energy from SFA with total MUFA was 1.06 (95\% CI $0.94,1.20$ ). Isoenergetically replacing 5\% of energy from SFA with P-MUFA or fried P-MUFA was associated with 16 and $24 \%$ higher risk of T2D, respectively, whereas the same replacement with A-MUFA did not show significant changes in T2D risk. Finally, T2D risk was $26 \%$ higher when 
isoenergetically substituting $5 \%$ of energy from P-MUFA for the sum of SFA and A-MUFA. The same substitution using fried P-MUFA was related to a $33 \%$ higher risk of T2D (online Supplementary Fig. S2).

\section{Sensitivity analyses}

In sensitivity analyses, the documented associations between MUFA consumption and T2D risk were not materially changed after further adjusting for the consumption of fruits and vegetables, dietary cholesterol or alternative healthy eating index and excluding the T2D cases within the first 3 years of follow-up (online Supplementary Tables S4-S7). Similar results were largely observed when we restricted the analysis to those with available data of blood glucose levels (online Supplementary Table S8).

\section{Subgroup analyses}

We detected a significant interaction between BMI and P-MUFA intake on the risk of T2D $\left(P_{\text {for interaction }}=0.0011\right)$. The positive association of P-MUFA with T2D was restricted to normal-weight participants $\left(P_{\text {trend }}=0.0002\right)$. The associations for A-MUFA intake with T2D risk were similar in all subgroups (online Supplementary Table S9).

\section{Discussion}

This prospective study is the first to assess the long-term associations of MUFA from different origins with the development of T2D. In this nationwide Chinese cohort, we found that intake of P-MUFA was positively associated with T2D risk, which was mainly driven by fried plant MUFA. The intake of MUFA from animal sources was not associated with T2D. T2D risk was higher when SFA or SFA combined with A-MUFA were isoenergetically replaced by fried P-MUFA.

Previous epidemiological data linking MUFA consumption to T2D development are conflicting. It has been speculated that the positive or null associations of total MUFA with T2D were attributed to the fact that MUFA and animal fat (SFA) were derived from shared food sources such as meat and dairy products in the Western diet and thus had a high correlation (online Supplementary Table S2). SFA and processed meat have been associated with higher T2D incidence in the Health Professionals Follow-up Study ${ }^{(16)}$, while replacing SFA with MUFA was related to lower risk in the Nurses' Health Study ${ }^{(15)}$. On the contrary, the major plant sources of MUFA in Western countries, olive oil ${ }^{(18,29)}$ and nuts ${ }^{(30,31)}$, have been correlated with a lower risk of T2D in multiple studies. Together, accumulating evidence indicates the importance of food sources of MUFA when evaluating the health benefit of MUFA.

Interventional studies supported the evidence of ameliorative effects of MUFA on glycaemic control and insulin resistance, wherein MUFA were from plant sources (e.g. olive oil, sunflower oil and rapeseed oil), compared with SFA or carbohydrates, and thus may not be confounded by animal fats ${ }^{(6-9)}$. However, when MUFA were differentiated from plant and animal sources, here we found that P-MUFA were unexpectedly and strongly related to a higher risk of T2D. In addition, replacing SFA with P-MUFA showed a significantly higher risk of T2D. These findings could be due to distinctive food sources of P-MUFA and cooking methods in China.

In the CHNS, major sources of P-MUFA were peanut oil, rapeseed oil and soyabean oil but not olive oil or nuts. Although rapeseed oil could improve glycaemic control in a randomised controlled trial ${ }^{(32)}$ and peanut oil was able to enhance insulin secretion in diabetic mice ${ }^{(33)}$, soyabean oil treatment presented insulin resistance in Wistar rats ${ }^{(34)}$. Importantly, these types of vegetable oils are mainly used for cooking but not for salad dressing, with stir-frying and griddling applied as usual preparation methods in China. Frying of vegetable oils may produce harmful changes, including the increase in energy density, NEFA contents, the ratio of hypocholesterolaemic:hypercholesterolaemic fatty acids and the formation of trans-fatty acids, TAG polymers and dimers ${ }^{(35,36)}$. In addition, lipid peroxidation products from frying process could induce inflammation ${ }^{(37)}$, which plays a crucial role in the pathogenesis of diabetes ${ }^{(38)}$. Fried food consumption was significantly associated with incident $\mathrm{T} 2 \mathrm{D}^{(39)}$.

In the present study, $80 \%$ of vegetable oils were used for frying, including deep-frying, stir-frying and griddling. Moreover, fried bread sticks, a Chinese traditional breakfast meal made from deep-frying of dough, is also a contributor to the intake of P-MUFA second to vegetable oils in the CHNS. Therefore, we further analysed the associations for P-MUFA from fried and nonfried foods and found a strong positive association between fried P-MUFA and T2D risk. Together, these results demonstrate that consumption of fried P-MUFA mainly from fried vegetable oils and fried bread sticks may elevate the risk of T2D in China.

Interestingly, we did not find significant associations of fried A-MUFA with T2D risk. MUFA from animal products may be more thermally stable due to the presence of a large amount of SFA that are relatively resistant to oxidation in animal foods, whereas MUFA from plant foods with a high degree of unsaturation are susceptible to oxidation or peroxidation when exposed to heat and $\mathrm{O}_{2}{ }^{(40)}$. Compared with other cooking oils, olive oil is more thermally stable and less prone to oxidation ${ }^{(39,41)}$. The use of olive oil for frying was associated with a lower level of insulin resistance in a cross-sectional study ${ }^{(42)}$. However, olive oil consumption was rare in China during the present study period.

Few prospective studies have assessed the associations of individual MUFA like OA and PA with T2D risk. Dietary intake of OA, the primary MUFA from diet sources, showed neutral effects on T2D development in previous Western studies ${ }^{(13)}$. Substituting OA for SFA exhibited beneficial effects on insulin sensitivity through exerting anti-inflammatory activity, inhibiting endoplasmic reticulum stress and improving $\beta$-cell survival and insulin signalling ${ }^{(43)}$. However, a gradient of T2D risk was apparent with an increasing intake of OA in our study. For the putative mechanism, treatment with a high level of OA was able to induce hyperinsulinaemia which may be a primary event in the pathogenesis underlying T2D in the previous in vitro study ${ }^{(44)}$.

With regard to PA, the Cardiovascular Health Study reported no association of PA intake with T2D risk ${ }^{(13)}$, while the positive association for PA was attenuated to a null relationship when BMI and waist:hip ratio were further adjusted in an Australian study ${ }^{(45)}$. However, we found a strong inverse relationship 
between PA intake and T2D risk. In line with our findings, plasma phospholipid trans-palmitoleate, a distinctive biomarker of exogenous PA, was independently related to lower insulin resistance and incident diabetes in US adults ${ }^{(46)}$. Besides, PA could improve skeletal muscle insulin response, pancreatic $\beta$-cell survival, insulin secretion and adipocyte metabolism ${ }^{(47)}$ in vitro and ameliorate hyperglycaemia and insulin sensitivity in vivo ${ }^{(48)}$.

When further separating OA and PA from plant and animal sources, the consistent findings for plant-derived OA, plantderived PA and P-MUFA demonstrated that MUFA from plantbased foods were responsible for their promotion effects on T2D development. Moreover, the strong negative association with incident T2D for total PA was driven by animal-derived PA. Since the trace amount of PA was found in animal foods, residual confounding by other constituents could be possible and further corroboration of our results in larger studies is needed.

The strengths of our study included the nationally representative sample of the Chinese population, long follow-up duration and the cumulative average measurement of repeated MUFA intakes to reflect long-term diet. Since T2D cases might appear at baseline, we excluded incident T2D cases occurred during the initial 3 years of follow-up to further control for reverse causation and found the results unchanged. The present study also has some limitations. First, T2D incidence may be underestimated for those (49.0\% of all participants) with information only from questionnaire. In particular, participants with higher MUFA intake had higher socio-economic status and probably got better access to health care and thus might have fewer undiagnosed cases of diabetes. Nonetheless, when we restricted to those with available data of both plasma glucose and questionnaire information ( $n$ 7708), the positive associations of MUFA from plant sources with $\mathrm{T} 2 \mathrm{D}$ incidence remained significant. Second, we could not adjust for trans-fatty acid intake due to lack of data. Nevertheless, the overall trans-fatty acid consumption in China was very low and might not appreciably change the documented findings ${ }^{(49)}$. Third, although MUFA consumption was estimated using the combination of three consecutive $24-\mathrm{h}$ recalls and a weighing technique, measurement error was still inevitable which might have attenuated the observed associations due to the prospective design. Moreover, the distinctive Chinese dietary pattern and cooking methods might limit the generalisability of currently established findings to other populations. Finally, reverse causation could not be ruled out because the observational nature and unmeasured and residual confounding, such as family history of diabetes, adipose tissue mass and distribution, glycaemic index and glycaemic load, were still possible even after full adjustment.

In conclusion, the intakes of MUFA from animal and plant origins were divergently associated with T2D risk. Replacing SFA with fried P-MUFA but not A-MUFA was associated with a higher risk of T2D. Our findings suggested a potential detrimental effect of MUFA from fried plant sources, such as fried vegetable oils and fried bread sticks, on the prevalence of T2D among the Chinese population.

\section{Acknowledgements}

We thank the National Institute for Nutrition and Health, China Center for Disease Control and Prevention.

This work was supported by the National Natural Science Foundation of China (grant number 81773419), Chinese Institute of Nutrition DSM Research Fund (grant number CNSDSM-2017-035) and China National Program for Support of Top-Notch Young Professionals. This research uses data from the CHNS. We are grateful to research grant funding from the National Institute for Health, the Eunice Kennedy Shriver National Institute of Child Health and Human Development (R01 HD30880; P2C HD050924), the National Institute of Diabetes and Digestive and Kidney Diseases (R01 DK104371), the National Institute for Health Fogarty D43 TW009077 for financial support for the CHNS data collection and analysis files since 1989, the China-Japan Friendship Hospital, Ministry of Health for support for CHNS 2009, Chinese National Human Genome Center at Shanghai since 2009 and Beijing Municipal Center for Disease Prevention and Control since 2011. This research was supported by the National Natural Science Foundation of China (grant no. 81773419) and Chinese Institute of Nutrition DSM Research Fund (CNS-DSM-2017-035). The funders had no role in the design, analysis or writing of this article.

P. Z., Y. Z. and J. J. designed the study; P. Z., L. M., F. W. and L. C. provided statistical expertise; P. Z. wrote the manuscript; J. J. had full access to all of the data in the study and took responsibility for the integrity of the data and the accuracy of the data analysis; and all authors conducted the research, contributed to the interpretation of the results and critical revision of the manuscript for important intellectual content and read and approved the final manuscript.

The authors declare that they have no conflicts of interest.

\section{Supplementary material}

For supplementary material referred to in this article, please visit https://doi.org/10.1017/S0007114520000677

\section{References}

1. International Diabetes Federation (2017) IDF Diabetes Atlas. Brussels: International Diabetes Federation.

2. Pan XR, Yang WY, Li GW, et al. (1997) Prevalence of diabetes and its risk factors in China, 1994. National Diabetes Prevention and Control Cooperative Group. Diabetes Care 20, 1664-1669.

3. Wang L, Gao P, Zhang M, et al. (2017) Prevalence and ethnic pattern of diabetes and prediabetes in China in 2013. JAMA 317, 2515-2523.

4. Ley SH, Hamdy O, Mohan V, et al. (2014) Prevention and management of type 2 diabetes: dietary components and nutritional strategies. Lancet 383, 1999-2007.

5. Pablo P-M, Antonio G-R, Javier D-L, et al. (2011) Mediterranean diet rich in olive oil and obesity, metabolic syndrome and diabetes mellitus. Curr Pharm Design 17, 769-777.

6. Schwingshackl L, Strasser B \& Hoffmann G (2011) Effects of monounsaturated fatty acids on glycaemic control in patients with abnormal glucose metabolism: a systematic review and meta-analysis. Ann Nutr Metab 58, 290-296. 
7. Qian F, Korat AA, Malik V, et al. (2016) Metabolic effects of monounsaturated fatty acid-enriched diets compared with carbohydrate or polyunsaturated fatty acid-enriched diets in patients with type 2 diabetes: a systematic review and meta-analysis of randomized controlled trials. Diabetes Care 39, 1448-1457.

8. Vessby B, Uusitupa M, Hermansen K, et al. (2001) Substituting dietary saturated for monounsaturated fat impairs insulin sensitivity in healthy men and women: the KANWU study. Diabetologia 44, 312-319.

9. Pérez-Jiménez F, López-Miranda J, Pinillos MD, et al. (2001) A Mediterranean and a high-carbohydrate diet improve glucose metabolism in healthy young persons. Diabetologia $\mathbf{4 4}$ 2038-2043.

10. Mirmiran P, Esfandyari S, Moghadam SK, et al. (2018) Fatty acid quality and quantity of diet and risk of type 2 diabetes in adults: Tehran Lipid and Glucose Study. J Diabetes Complicat 32 655-659.

11. Guasch-Ferré M, Becerra-Tomás N, Ruiz-Canela M, et al. (2017) Total and subtypes of dietary fat intake and risk of type 2 diabetes mellitus in the Prevención con Dieta Mediterránea (PREDIMED) study. Am J Clin Nutr 105, 723-735.

12. Harding A-H, Day NE, Khaw K-T, et al. (2004) Dietary fat and the risk of clinical type 2 diabetes: the European Prospective Investigation of Cancer-Norfolk Study. Am J Epidemiol 159, 73-82.

13. Ma W, Wu JHY, Wang Q, et al. (2015) Prospective association of fatty acids in the de novo lipogenesis pathway with risk of type 2 diabetes: the Cardiovascular Health Study. Am J Clin Nutr 101, 153-163.

14. Meyer KA, Kushi LH, Jacobs DR, et al. (2001) Dietary fat and incidence of type 2 diabetes in older Iowa women. Diabetes Care 24, 1528.

15. Salmerón J, Hu FB, Manson JE, et al. (2001) Dietary fat intake and risk of type 2 diabetes in women. Am J Clin Nutr 73, 1019-1026.

16. van Dam RM, Willett WC, Rimm EB, et al. (2002) Dietary fat and meat intake in relation to risk of type 2 diabetes in men. Diabetes Care 25, 417

17. Alhazmi A, Stojanovski E, McEvoy M, et al. (2013) Macronutrient intake and type 2 diabetes risk in middle-aged Australian women. Results from the Australian Longitudinal Study on Women's Health. Public Health Nutr 17, 1587-1594

18. Schwingshackl L, Lampousi AM, Portillo MP, et al. (2017) Olive oil in the prevention and management of type 2 diabetes mellitus: a systematic review and meta-analysis of cohort studies and intervention trials. Nutr Diabetes 7, e262.

19. Risérus U, Willett WC \& Hu FB (2009) Dietary fats and prevention of type 2 diabetes. Prog Lipid Res $\mathbf{4 8}, 44-51$.

20. Popkin BM, Du S, Zhai F, et al. (2010) Cohort Profile: the China Health and Nutrition Survey - monitoring and understanding socio-economic and health change in China, 1989-2011. Int J Epidemiol 39, 1435-1440.

21. Popkin BM, Lu B \& Zhai F (2006) Understanding the nutrition transition: measuring rapid dietary changes in transitional countries. Public Health Nutr 5, 947-953.

22. Tee ES (2011) Development and promotion of Malaysian Dietary Guidelines. Asia Pac J Clin Nutr 20, 455-461.

23. China CDC (1991) Food Nutrient Components Table. Beijing. People's Medical Publishing House.

24. Institute of Nutrition and Food Safety (2002) China CDC: China Food Composition 2002. Beijing: Peking University Medical Press

25. Institute of Nutrition and Food Safety (2005) China CDC: China Food Composition 2004. Beijing: Peking University Medical Press.

26. Yao M, McCrory MA, Ma G, et al. (2003) Relative influence of diet and physical activity on body composition in urban Chinese adults. Am J Clin Nutr 77, 1409-1416.
27. Kulldorff M, Sinha R, Chow W-H, et al. (2000) Comparing odds ratios for nested subsets of dietary components. Int J Epidemiol 29, 1060-1064.

28. Wang Z, Adair LS, Cai J, et al. (2017) Diet quality is linked to insulin resistance among adults in China. J Nutr 147, 2102-2108.

29. Guasch-Ferré M, Hruby A, Salas-Salvadó J, et al. (2015) Olive oil consumption and risk of type 2 diabetes in US women. Am J Clin Nutr 102, 479-486.

30. Villegas R, Gao Y-T, Yang G, et al. (2008) Legume and soy food intake and the incidence of type 2 diabetes in the Shanghai Women's Health Study. Am J Clin Nutr 87, 162-167.

31. Jiang R, Manson JE, Stampfer MJ, et al. (2002) Nut and peanut butter consumption and risk of type 2 diabetes in women. JAMA 288, 2554-2560.

32. Jenkins DJA, Kendall CWC, Vuksan V, et al. (2014) Effect of lowering the glycemic load with canola oil on glycemic control and cardiovascular risk factors: a randomized controlled trial. Diabetes Care 37, 1806.

33. Vassiliou EK, Gonzalez A, Garcia C, et al. (2009) Oleic acid and peanut oil high in oleic acid reverse the inhibitory effect of insulin production of the inflammatory cytokine TNF- $\alpha$ both in vitro and in vivo systems. Lipids Health Dis $\mathbf{8}, 25-25$.

34. Nunes E, Peixoto F, Louro T, et al. (2007) Soybean oil treatment impairs glucose-stimulated insulin secretion and changes fatty acid composition of normal and diabetic islets. Acta Diabetol 44, 121-130.

35. Narayanankutty A, Manalil JJ, Suseela IM, et al. (2016) Deep fried edible oils disturb hepatic redox equilibrium and heightens lipotoxicity and hepatosteatosis in male Wistar rats. Hum Exp Toxicol 36, 919-930.

36. Sayon-Orea C, Carlos S \& Martínez-Gonzalez MA (2015) Does cooking with vegetable oils increase the risk of chronic diseases?: a systematic review. Br J Nutr 113, S36-S48.

37. Miller YI \& Shyy JYJ (2017) Context-dependent role of oxidized lipids and lipoproteins in inflammation. Trends Endocrinol Metab 28, 143-152.

38. Olefsky JM \& Glass CK (2010) Macrophages, inflammation, and insulin resistance. Annu Rev Physiol 72, 219-246.

39. Cahill LE, Pan A, Chiuve SE, et al. (2014) Fried-food consumption and risk of type 2 diabetes and coronary artery disease: a prospective study in 2 cohorts of US women and men. Am JClin Nutr 100, 667-675.

40. Shahidi F \& Zhong Y (2010) Lipid oxidation and improving the oxidative stability. Chem Soc Rev 39, 4067-4079.

41. Zotos A, Kotaras A \& Mikras E (2012) Effect of baking of sardine (Sardina pilchardus) and frying of anchovy (Engraulis encrasicholus) in olive and sunflower oil on their quality. Food Sci Technol Int 19, 11-23.

42. Soriguer F, Esteva I, Rojo-Martinez G, et al. (2004) Oleic acid from cooking oils is associated with lower insulin resistance in the general population (Pizarra study). Eur J Endocrinol 150, 33-39.

43. Palomer X, Pizarro-Delgado J, Barroso E, et al. (2018) Palmitic and oleic acid: the Yin and Yang of fatty acids in type 2 diabetes mellitus. Trends Endocrinol Metab 29, 178-190.

44. Zhang Y, Xiao M, Niu G, et al. (2005) Mechanisms of oleic acid deterioration in insulin secretion: role in the pathogenesis of type 2 diabetes. Life Sci 77, 2071-2081.

45. Hodge AM, English DR, O'Dea K, et al. (2007) Plasma phospholipid and dietary fatty acids as predictors of type 2 diabetes: interpreting the role of linoleic acid. Am J Clin Nutr 86, 189-197.

46. Mozaffarian D, Cao H, King IB, et al. (2010) Trans-palmitoleic acid, metabolic risk factors, and new-onset diabetes in US adults. Ann Intern Med 153, 790-799. 
47. Everson Araujo N \& Alex R (2017) Implications of palmitoleic acid (palmitoleate) on glucose homeostasis, insulin resistance and diabetes. Curr Drug Targets 18, 619-628.

48. Yang Z-H, Miyahara H \& Hatanaka A (2011) Chronic administration of palmitoleic acid reduces insulin resistance and hepatic lipid accumulation in KK- $\mathrm{A}^{\mathrm{y}}$ mice with genetic type 2 diabetes. Lipids Health Dis 10, 120.

49. Yu DX, Sun Q, Ye XW, et al. (2012) Erythrocyte trans-fatty acids, type 2 diabetes and cardiovascular risk factors in middle-aged and older Chinese individuals. Diabetologia 55, 2954-2962. 